\title{
Gain estimate for exoplanet detection with adaptive optics
}

\author{
V.F. Canales and M.P. Cagigal \\ Dpto. de Fisica Aplicada, Universidad de Cantabria, Avda. Castros S/N, 39005 Santander, Spain
}

Received June 9; accepted June 9, 2000

\begin{abstract}
Atmospheric turbulence imposes the resolution limit attainable by large ground-based telescopes. Adaptive optics systems can compensate turbulenceinduced distortions. One of the most difficult task for telescopes assisted by adaptive optics systems is the detection of extrasolar planets since a compensation level higher than in other applications is needed. In this context, the gain of the system (ratio between the peak and the halo intensity) gives a good description of the system performance. A way to theoretically estimate the system gain and a technique to estimate it from the measurement of the experimental Strehl ratio are developed using a single model of the PSF of compensated wavefronts. From it, the integration time required to detect a Jupiter-like planet is derived from the number of system actuators and the atmospheric conditions.
\end{abstract}

Key words: atmospheric effects — techniques: image processing — telescopes

\section{Introduction}

Random aberrations on the pupil of the telescope due to atmospheric turbulence determine the angular resolution of ground-based telescopes. Adaptive optics systems perform a real time correction of the degradation introduced by the atmosphere (Wang \& Markey 1978; Rigaut et al. 1991; Roggemann 1991). A wavefront sensor measures the instantaneous aberrations and, using this information, a deformable mirror changes its shape to compensate this distortion. One of the most interesting subjects in astronomy is the use of adaptive optics systems to detect extrasolar planets. A series of important problems appears in this kind of detection. The main limitation is the brightness difference between the star and the planet (about $10^{9}$ times). After compensation, the difference between

Send offprint requests to: V.F. Canales,

e-mail: mpc@opt.unican.es the star halo and the peak of the planet is expected to be about $10^{4}$. In actual systems to reduce the star-planet luminosity ratio to this limit, it would be necessary to perform a compensation as complete as possible. The ideal high gain required for exoplanet detection is limited by a number of noises and technical difficulties. Papers of Angel (1994) and Stahl \& Sandler (1995) show these problems and some possible solutions. Then it is necessary to reduce the scattered light of the star and suppress the diffracted light (Nakajima 1994). This compensation will reduce the long integration time necessary to increase the contrast between planet and background.

The improvement of the adaptive optics system on the brightness difference is represented by the system gain that is defined as the ratio between the peak and halo intensities. This parameter establishes a good connection between the system performances and the detection requirements (Labeyrie 1995). In this paper we introduce a theoretical expression for the system gain. The procedure we propose to obtain the gain is based on the image formation process (Cagigal \& Canales 2000a; Goodman 1985). The wavefront is described through the structure function which provides two parameters: the correlation length and the residual phase variance in the compensated wavefront. From them it is easy to develop a model to describe the point spread function (PSF) of the system, so that, a clear relationship can be established between the PSF halo and the parameters obtained from the structure function. The gain expression we obtain resembles the approximated one proposed by Angel (1994). The main difference is that our expression has a more complex dependence on the residual phase variance, consequence of the procedure followed to obtain it. Under a simple approximation both expressions are equivalent since the correlation length and the actuator size equate for high compensation level. Hence, we propose a theoretical procedure based on the image formation process that allows an accurate estimate of the system gain using only two parameters: the correlation length of the compensated wavefront and the residual phase variance. Furthermore, we show an experimental method 
to estimate the actual residual phase variance in the compensated wavefront, which allows us to obtain a more realistic estimation of the system gain. Still there are some sources of errors that are not taken into account, as scintillation or residual temporal decorrelation errors, which would require a deeper analysis.

\section{Phase screen behaviour}

This section describes the wavefront decomposition into Zernike polynomials, the phase structure function of the wavefront, the generalization of the Fried parameter and the phase correlation length behavior.

\subsection{Wavefront description}

Optical wavefronts are two dimensional functions that can be decomposed into Zernike polynomials which are separable in angle and radius and form an orthogonal basis. We will use the definition given by Noll (1976):

$\phi(r, \theta)=\sum_{i=1}^{\infty} a_{i} Z_{i}(r, \theta)$

where $a_{i}$ are the coefficients of the corresponding Zernike polynomials $\left(Z_{i}\right)$. The effect of partial compensation on the wavefront is that some of the decomposition coefficients vanish. The residual distortion in the compensated wavefront may be estimated using the Noll expression for the average variance over the wavefront surface once the first $j$ Zernike terms have been corrected:

$\Delta_{j}=\sum_{i=j+1}^{\infty}\left\langle\left|a_{i}^{2}\right|\right\rangle=\operatorname{coef}(j)\left(\frac{D}{r_{0}}\right)^{5 / 3}$

where \langle\rangle denotes an ensemble average and $\operatorname{coef}(j)$ is the corresponding coefficient given by Noll. The maximum number of modes that can be corrected is related with the number of actuators in the system.

\subsection{Structure function}

The phase structure function is defined as:

$D_{\phi}\left(\boldsymbol{r}-\boldsymbol{r}^{\prime}\right)=\left\langle\left[\phi(\boldsymbol{r})-\phi\left(\boldsymbol{r}^{\prime}\right)\right]^{2}\right\rangle$.

For apertures over $1 \mathrm{~m}$, even in the best seeing sites, the phase fluctuation has a Gaussian distribution because of the central limit theorem. Its mean value is zero and, hence, it is fully described by its second moment. The structure function being the second moment for differential phase fluctuation, completely specifies the spatial statistics of phase fluctuation. Based on the Kolmogorov (1961) theory of turbulence, it is possible to show that in the absence of compensation the phase structure function may be written as:

$D_{\phi}(r)=6.88\left(\frac{r}{r_{0}}\right)^{5 / 3}$ where $r_{0}$ is the Fried parameter (Fried 1965). It is known (Roddier 1990) that the shape of the structure function varies as a function of the compensation degree. As the separation between points becomes arbitrarily large the autocorrelation function tends to zero and the structure function saturates to $2 \Delta_{j}$. However, for small separation distances the curve still follows the $5 / 3$ power law (Rigaut et al. 1997; Hardy 1998) and can be fitted using the following expression:

$D_{\phi}(r)=6.88\left(\frac{r}{\rho_{0}}\right)^{5 / 3}$

where the value of the parameter $\rho_{0}$, the generalized Fried parameter, increases with the compensation degree. Figure 1 shows this behavior of the structure function for uncompensated wave fronts (short-dashed curve) obtained from simulation, and for 6 (solid curve), 21 (circles), 41 (long-dashed curve) and 81 (triangles) corrected modes. This behaviour of the structure function is not restricted to those systems that correct pure Zernike modes. A first region described by Eq. (5) that saturates to $2 \Delta_{j}$ for distances greater than the correlation length has been found in noisy systems and in curvature-based systems (Cagigal \& Canales 2000a).

\subsection{Correlation length}

The phase correlation length in the wavefront, $l_{\text {corr }}$, can be obtained from the structure function (Valley \& Wandzura 1979). It is defined as the distance value for which the structure function leaves the $5 / 3$ power behavior and reaches the constant value $2 \Delta_{j}$. In this point it is fulfilled that:

$6.88\left(\frac{l_{\text {corr }}}{\rho_{0}}\right)^{5 / 3}=2 \Delta_{j}$.

It is interesting to note that the parameters $\Delta_{j}$ and $\rho_{0}$ are determined by the number of corrected polynomials and by the value of the ratio $D / r_{0}$. However, the correlation length is completely determined by the number of corrected polynomials (in $D$ units) as it can be deduced from previous works (Valley \& Wandzura 1979). The behaviour of $l_{\text {corr }}$ was described in a previous paper (Cagigal \& Canales 2000a) by fitting a generic curve to the values obtained from the simulated structure functions. The fitted curve is given by:

$l_{\text {corr }} \approx 0.286 j^{-0.362} D$

where $j$ is the number of corrected polynomials, that we relate to the number of system actuators. We have checked that this is a robust curve under deviations from an ideal compensation process.

\section{Gain of the adaptive optics system}

The Fried parameter gives a measure of the maximum resolution attainable in uncompensated long-exposure 


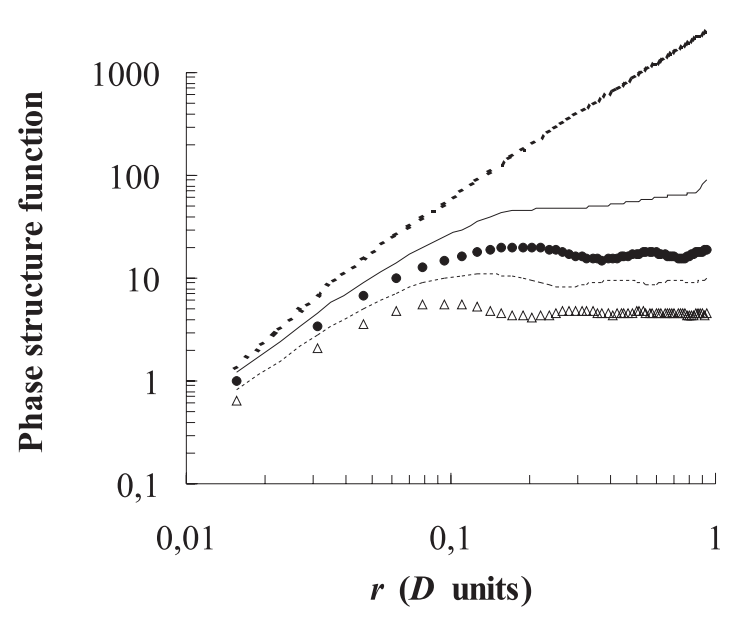

Fig. 1. Structure function of the wavefront phase for $D / r_{0}=$ 38.4 and 1 (short-dashed curve), 6 (solid curve), 21 (circles), 41 (long dashed curve) and 81 (triangles) corrected modes

images. Thus, the point spread function (PSF) size is proportional to $\lambda / r_{0}$. As compensation increases the image becomes a bright core surrounded by a speckled halo (Goodman 1985; Hardy 1998; Smithson et al. 1988). The halo width, $\omega_{0}$, can be obtained, equivalently to Eq. (7.20) in Roddier (1981), from:

$$
\int_{\text {image }} I_{\text {halo }}(\boldsymbol{x}) \mathrm{d} \boldsymbol{x}=\frac{\pi}{4} \omega_{0}^{2} I_{\text {halo }}(\mathbf{0}) \text {. }
$$

The left part of this equation is the total energy at the halo. To estimate its value, we will use the following approximated expression for the optical transfer function (Goodman 1985)

$$
\begin{aligned}
\mathrm{OTF}= & \mathrm{OTF}_{\mathrm{TEL}} \\
& \times\left[\exp \left(-\Delta_{j}\right)+\exp \left(-\Delta_{j}\right)\left(\exp \left(\gamma \Delta_{j}\right)-1\right)\right]
\end{aligned}
$$

As the separation between points becomes arbitrarily large, the autocorrelation function $\gamma$ tends to zero and, hence, the first term is the asymptote to which the OTF falls, whereas the second represents the rise above that asymptote. Assuming that the OTF of the original telescope is much wider than the second term, an approximated expression for the PSF becomes:

$$
\begin{aligned}
\mathrm{PSF}= & \mathrm{PSF}_{\mathrm{TEL}} \exp \left(-\Delta_{j}\right) \\
& +\mathcal{F}\left[\exp \left(-\Delta_{j}\right)\left(\exp \left(\gamma \Delta_{j}\right)-1\right)\right] .
\end{aligned}
$$

The first term (coherent energy) can be interpreted as a diffraction-limited core of the PSF and the second a much broader halo. Thus, the coherent energy is approximately a fraction $\exp \left(-\Delta_{j}\right)$ of the total energy, and, consequently, the total energy in the halo is $1-\exp \left(-\Delta_{j}\right)$ times the total energy, $E_{\mathrm{T}}$. Then the first term of Eq. (8) can be estimated as:

$$
\int_{\text {image }} I_{\text {halo }}(\boldsymbol{x}) \mathrm{d} \boldsymbol{x}=E_{\mathrm{T}}\left[1-\exp \left(-\Delta_{j}\right)\right] \text {. }
$$

On the other hand, for high degrees of correction (phase error lower than $1 \mathrm{rad} \mathrm{rms}) \omega_{0}=\lambda f / 2 l_{\text {corr }}$ is the average diameter of the halo (where $f$ is the focal length of the system). Hence, the halo mean intensity $\left(I_{\mathrm{h}}\right)$ can be estimated as the ratio between the total energy in the halo $E_{\mathrm{T}}\left[1-\exp \left(-\Delta_{j}\right)\right]$ and the halo area:

$I_{\mathrm{h}}=\frac{E_{\mathrm{T}}\left[1-\exp \left(-\Delta_{j}\right)\right]}{\pi\left(\lambda f / 2 l_{\text {corr }}\right)^{2}}$.

Then the mean intensity in the coherent peak is estimated as:

$I_{\mathrm{c}}=\frac{E_{\mathrm{T}} \exp \left(-\Delta_{j}\right)}{\pi(\lambda f / 2 D)^{2}}$.

Now, in an experiment where the energy incoming from the star is $E_{*}$ and the energy from the planet $E_{\mathrm{o}}$, the ratio between the intensity at the star and the peak of the planet will be:

$$
\frac{R}{G}=\frac{E_{*}\left[1-\exp \left(-\Delta_{j}\right)\right]}{\pi\left(\lambda f / 2 l_{\text {corr }}\right)^{2}} \frac{\pi(\lambda f / 2 D)^{2}}{E_{\mathrm{o}} \exp \left(-\Delta_{j}\right)}
$$

where $R=E_{*} / E_{\mathrm{o}}$ and $G$ is the gain of the adaptive optics. Now, $G$ can be written as an explicit function of the residual phase variance and $l_{\text {corr }}$ as:

$G=\frac{\exp \left(-\Delta_{j}\right)}{1-\exp \left(-\Delta_{j}\right)}\left(\frac{D}{l_{\text {corr }}}\right)^{2}$.

Hence, this expression allows us to estimate the gain of the adaptive system using the theoretical values of the residual phase variance $\Delta_{j}$ and of the correlation length. It can be approximated by:

$G \approx \frac{1}{\Delta_{j}}\left(\frac{D}{l_{\text {corr }}}\right)^{2}$.

This approximated expression is similar to that obtained by Angel (1994) $G \approx\left(1 / \Delta_{j}\right)\left[(D / \Delta x)^{2}\right]$. The main difference is that we determine the halo width from the correlation length $l_{\text {corr }}$, instead of $\Delta x$, which is the projection of the actuator size over the telescope aperture. However, in the high correction range that is considered in this paper, $l_{\text {corr }}$ and $\Delta x$ have identical evolutions and represent the greatest scale in which turbulence is corrected. Figure 2 shows how the gain evolves as a function of the degree of compensation for a series of $D / r_{0}$ values. It can be seen that values obtained using Eq. (15) are slightly lower than those predicted by Angel's expression. Our method is more accurate because it is derived from the PSF model, instead of an approximate estimate.

Finally, we will compare the integration time required using gain values derived from Eq. (15) with that obtained by Angel. The comparison will be performed considering the ideal compensation system proposed by Angel (1994). The compensation system uses $10^{4}$ actuators at a frequency higher than $2 \mathrm{kHz}$ and scintillation is not considered (it is assumed that it has been previously compensated). From the Angel's expression relating the integration time $T$, the signal-to-noise ratio $S / N$ and the system 


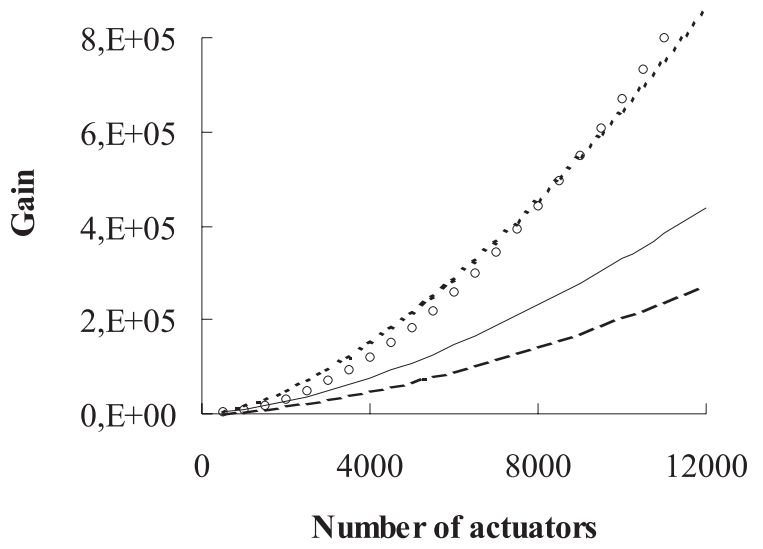

Fig. 2. System gain as a function of the number of actuators for $D / r_{0}=20$ (short-dashed curve), 30 (solid curve) and 40 (long-dashed curve). Values obtained by Angel, for $D / r_{0}=20$, are also shown for comparison (dots)

gain, it is possible to obtain an expression for the integration time as a function of the residual phase variance:

$T=\Delta t_{\mathrm{opt}} \frac{S}{N} \frac{R^{2}}{G^{2}}$

where the system gain $G$ can be estimated from Eqs. (15) or (16). A typical value of the optimal duration of the adaptive correction is $\Delta t_{\mathrm{opt}}=0.35 \mathrm{~ms}$. We will consider that the planet is detectable for a $S / N=5$ and the planet luminosity is $10^{9}$ times that of the star. Figure 3 shows the exposure time evolution as a function of the degree of compensation for a series of $D / r_{0}$ values. The integration time is in order of magnitude similar to that predicted by Angel. As an example with a telescope of $3 \mathrm{~m}$ and a Fried parameter of $10 \mathrm{~cm}$ a planet could be detected in a 3 hours integration. These values are too optimistic because an ideal compensation process is considered.

In actual experiments the residual phase variance can be different from that theoretically expected due to a series of noises. In this case, the actual phase variance can be obtained from the experimental estimate of the Strehl ratio either using the Marechal approximation $1-\sigma_{\phi}^{2} \approx S R$, which provides quite good estimates for high compensation levels, or resolving a more accurate expression recently proposed (Cagigal \& Canales 2000b)

$\sigma_{\phi}^{2}=3.44\left\{0.286 j^{-0.362}\left[\frac{S R-\exp \left(-\sigma_{\phi}^{2}\right)}{1-\exp \left(-\sigma_{\phi}^{2}\right)}\right]^{-1 / 2}\right\}^{5 / 3}$.

This expression has the advantage that it remains valid for the whole range of compensation degrees. Hence the method to obtain the system gain will provide good results even in noisy conditions. Still there are some error sources not taken into account as scintillation or residual temporal decorrelation errors, that would require a more detailed analysis.

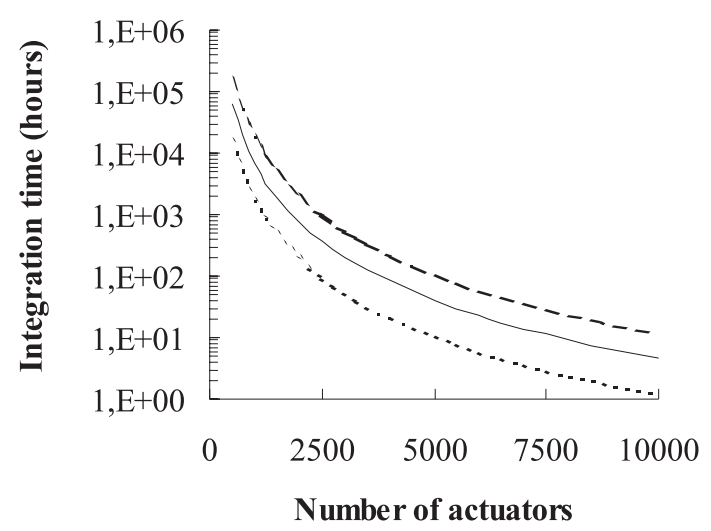

Fig. 3. Integration time to give an $S / N$ ratio of 5 for $R=10^{9}$ and $D / r_{0}=20$ (short-dashed curve), 30 (solid curve) and 40 (long-dashed curve)

\section{Conclusions}

Starting from a simple model of the phase screen behavior we have developed an expression for the PSF of a telescope using an adaptive optics system. From this model it is easy to obtain the system gain as a function of the residual phase variance and the correlation length. This procedure can be applicable even if there are relevant error sources involved in the compensation process, using experimental estimates of the phase variance. Finally, as a consequence of the influence of the system gain over the integration time, we obtain an integration time for direct exoplanet detection similar to that previously estimated in other references, but applicable in more realistic conditions, though some effort is still required to obtain a complete analysis.

Acknowledgements. This work has been supported by the Dirección General de Ensenanza Superior e Investigación Científica under project: PB97-0355.

\section{References}

Angel J.R.P., 1994, Nat 368, 203

Cagigal M.P., Canales V.F., 2000a, J. Opt. Soc. Am. A. 17, 903

Cagigal M.P., Canales V.F., 2000b, J. Opt. Soc. Am. A 17, 1312

Conan J., 1995, Étude de la correction partielle en optique adaptative, Ph.D. dissertation, Office Nationale d'Études et de Recherces Aérospatiales, Pub. 1995-1, Paris

Fried D.L., 1965, J. Opt. Soc. Am. 55, 1427

Goodman J.W., 1985, Statistical optics. Wiley-Interscience, New York

Hardy J.W., 1998, Adaptive Optics for Astronomical Telescopes. Oxford University Press, New York

Kolmogorov A., 1961, Classic papers on statistical theory, Friedlander S., Topper L. (eds.). Interscience Publishers, Inc., New York 
Labeyrie, 1995, A\&A 298, 544

Nakajima T., 1994, ApJ 425, 348

Noll R.J., 1976, J. Opt. Soc. Am. 66, 207

Rigaut F., Rousset G., Fontanella J.C., et al., 1991, A\&A 250, 280

Rigaut F., Ellerbroek B.L., Northcott M.J., 1997, Appl. Opt. 36,2856

Roddier F., 1981, The effects of atmospheric turbulence in optical astronomy, in Progress in Optics XIX, Wolf E. (ed.).
North Holland, Amsterdam

Roddier N., 1990, Opt. Eng. 29, 1174

Roggemann M.C., 1991, Appl. Opt. 30, 4227

Roggemann M.C., Welsh B., 1996, Imaging through turbulence. CRC Press, Boca Raton, Florida

Stahl S.M., Sandler D.G., 1995, ApJ 454, L153

Smithson R., Peri M., Benson R., 1988, App. Opt. 27, 1615

Wang J.Y., Markey J.K., 1978, J. Opt. Soc. Am. 68, 78

Valley G., Wandzura S., 1979, J. Opt. Soc. Am. 69, 712 